\title{
Existence of Solutions for Riemann-Liouville Fractional Boundary Value Problem
}

\author{
Wenzhe Xie, ${ }^{1}$ Jing Xiao, ${ }^{2}$ and Zhiguo Luo ${ }^{1}$ \\ ${ }^{1}$ Department of Mathematics, Hunan Normal University, Changsha, Hunan 410081, China \\ ${ }^{2}$ Department of Information Engineering, Guangdong Medical College, Dongguan, Guangdong 523808, China
}

Correspondence should be addressed to Jing Xiao; xjazyh@163.com

Received 8 May 2014; Revised 29 June 2014; Accepted 1 July 2014; Published 15 July 2014

Academic Editor: Ali H. Bhrawy

Copyright (c) 2014 Wenzhe Xie et al. This is an open access article distributed under the Creative Commons Attribution License, which permits unrestricted use, distribution, and reproduction in any medium, provided the original work is properly cited.

By using the method of upper and lower solutions and fixed point theorems, the existence of solutions for a Riemann-Liouville fractional boundary value problem with the nonlinear term depending on fractional derivative of lower order is obtained under the classical Nagumo conditions. Also, some results concerning Riemann-Liouville fractional derivative at extreme points are established with weaker hypotheses, which improve some works in Al-Refai (2012). As applications, an example is presented to illustrate our main results.

\section{Introduction}

Fractional calculus, which is a powerful tool for the description of memory and hereditary properties of materials and processes, has attracted the attention of many researchers and has been successfully applied in various fields, such as economics, engineering, and physical sciences. For the development of the theory of fractional calculus, we refer the readers to the monographs $[1,2]$ and references therein.

Recently, some papers have dealt with the existence of the solutions for fractional boundary value problems mainly by means of fixed point theorems [3-5], Leray-Schauder continuation principle [6], critical point theory [7, 8], and the method of upper and lower solutions [9-12]. Therein, various kinds of boundary value problems for nonlinear fractional differential equations have been studied, and some excellent results have been established. We are particularly interested in the case where the nonlinear term depends explicitly on the fractional derivative of lower order. Specifically, Su and Zhang in [13] deal with a boundary value problem of a fractional differential equation with the nonlinear term dependent on a fractional derivative of lower order on the semi-infinite interval:

$$
\begin{array}{cc}
D_{0+}^{\alpha} u(t)+f\left(t, u(t), D_{0+}^{\alpha-1} u(t)\right)=0, & t \in[0,+\infty), \\
u(0)=0, \quad D_{0+}^{\alpha-1} u(\infty)=u_{\infty}, \quad u_{\infty} \in \mathbb{R},
\end{array}
$$

where $1<\alpha \leq 2, f \in C\left([0,+\infty) \times \mathbb{R}^{2}, \mathbb{R}\right)$, and $D_{0+}^{\alpha}$ and $D_{0+}^{\alpha-1}$ are the standard Riemann-Liouville fractional derivatives. The existence results for solutions are obtained by using Schauder's fixed point theorem on an unbounded domain. And in [14] Agarwal et al. investigate the existence of positive solutions for the singular Riemann-Liouville fractional Dirichlet boundary value problem:

$$
\begin{gathered}
D_{0+}^{\alpha} u(t)+f\left(t, u(t), D_{0+}^{\mu} u(t)\right)=0, \\
u(0)=u(1)=0,
\end{gathered}
$$

where $1<\alpha<2,0<\mu \leq \alpha-1$, and $f(t, x, y)>0$ satisfying the Carathéodory conditions and being singular at $x=0$. The proofs are based on a fixed point theorem on a cone, regularization, and sequential techniques.

It is well known that the method of upper and lower solutions is a powerful tool for proving the existence and multiplicity results of solutions for nonlinear differential equations. Using this method and monotone iterative technique, the authors in [10-12] investigate some nonlinear fractional differential equations with nonlinear boundary conditions and establish some fractional comparison principles and further obtain the existence results of solutions, including extremal solutions, yet, mainly focus on the case of order $\delta \in(0,1)$. 
Nagumo conditions play an important role in the boundary value problems with nonlinear term involved in the derivative, since as it is known, for instance, for second order differential equations, the existence of upper and lower solutions, by itself, is not sufficient to ensure the existence of solutions. The studies dealing with the Nagumo conditions are well established by applying the method of upper and lower solutions combined with fixed point theorem or topological degree theory for the case of integer order (see [15-17]). To the best of our knowledge, no work has been done concerning the existence of solutions for fractional boundary value problem with nonlinear terms involving fractional derivative under Nagumo conditions.

Inspirited by the papers mentioned above, in this paper, under Nagumo conditions we aim to apply the method of upper and lower solutions combined with fixed point theorems to discuss the existence of solutions for the following Riemann-Liouville fractional boundary value problem (FBVP for short):

$$
\begin{gathered}
D_{0+}^{q} u(t)+f\left(t, u(t), D_{0+}^{q-1} u(t)\right)=0, \quad t \in[0,1], \\
u(0)=0, \quad D_{0+}^{q-1} u(1)=\eta,
\end{gathered}
$$

where $f:[0,1] \times \mathbb{R}^{2} \rightarrow \mathbb{R}$ is continuous and $D_{0+}^{q}$ is the Riemann-Liouville fractional derivative of order $1<q<2$, $\eta \in \mathbb{R}$. Our results extend some classical results for second order differential equations to the case of fractional order $1<$ $q<2$.

This paper is organized as follows. In Section 2, some notations, definitions, and lemmas are presented. We establish some results concerning the Riemann-Liouville fractional derivatives at extreme points under weaker conditions than those in [18]. In Section 3, sufficient conditions are given for the existence of at least one solution for FBVP (3). In Section 4, an explicit example is given to illustrate our main results.

\section{Preliminaries}

In this section, we introduce some definitions and lemmas, which are used throughout this paper.

A function $f:[0,1] \rightarrow \mathbb{R}$ is Hölder continuous, if there exist nonnegative constant $K$ and exponent $\lambda \in(0,1)$, such that

$$
\left|f\left(t_{1}\right)-f\left(t_{2}\right)\right| \leq K\left|t_{1}-t_{2}\right|^{\lambda}, \quad t_{1}, t_{2} \in[0,1] .
$$

A function $f:[0,1] \rightarrow \mathbb{R}$ is, especially, Lipschitz continuous, if the above inequality holds for $\lambda=1$.

Definition 1 (see $[1,2]$ ). The Riemann-Liouville fractional integral of order $\delta>0$ of a function $f:[0, \infty) \rightarrow \mathbb{R}$ is given by

$$
I_{0+}^{\delta} f(t)=\frac{1}{\Gamma(\delta)} \int_{0}^{t} \frac{f(s)}{(t-s)^{1-\delta}} d s
$$

where $\Gamma(\cdot)$ is the gamma function, provided that the right side is pointwise defined on $[0, \infty)$.
Definition 2 (see $[1,2])$. The Riemann-Liouville fractional derivative of order $\delta>0$ of a function $f:[0, \infty) \rightarrow \mathbb{R}$ is given by

$$
\begin{array}{r}
D_{0+}^{\delta} f(t)=\frac{1}{\Gamma(n-\delta)}\left(\frac{d}{d t}\right)^{n} \int_{0}^{t} \frac{f(s)}{(t-s)^{\delta+1-n}} d s, \\
n-1<\delta<n,
\end{array}
$$

provided that the right side is pointwise defined on $[0, \infty)$.

Lemma 3 (see [2]). Suppose that $D_{0+}^{\delta} u(t)$ exists and is integrable on $[0,1], \delta \geq p \geq 0$; then

$$
\begin{array}{r}
I_{0+}^{p} D_{0+}^{\delta} u(t)=D_{0+}^{\delta-p} u(t)-\sum_{i=1}^{n} \frac{D_{0+}^{\delta-i} u(t)_{\mid t=0}}{\Gamma(p+1-i)} t^{p-i}, \\
t \in[0,1], \quad n=[\delta]+1 .
\end{array}
$$

Property 1 (see $[1,2])$. Let $\delta \geq p>0$. The following properties are well known:

(1) $I_{0+}^{\delta}:\left\{\begin{array}{l}C[0,1] \rightarrow C[0,1], \\ L(0,1) \rightarrow L(0,1),\end{array}\right.$

(2) $D_{0+}^{p} I_{0+}^{\delta} u(t)=I_{0+}^{\delta-p} u(t), t \in[0,1], u(t) \in L(0,1)$,

(3) $\lim _{\delta \rightarrow 0} I_{0+}^{\delta} u(t)=u(t), \forall t \in(0,1], u(t) \in C[0,1]$,

(4) $D_{0+}^{\delta+1} u(t)=D^{1} D_{0+}^{\delta} u(t)$, where $D^{1}=d / d t$, provided that $D_{0+}^{\delta+1} u(t)$ exists.

Lemma 4. Suppose that $u(t) \in C[0,1]$ and that $D_{0+}^{q} u(t) \in$ $L(0,1), q \in(1,2)$, then $\lim _{\delta \rightarrow q^{-}} D_{0+}^{\delta} u(t)=D_{0+}^{q} u(t), t \in(0,1]$.

Proof. For all $\delta \in(1, q], q \in(1,2)$, we let

$$
f(t):=D_{0+}^{q} u(t), \quad F(t):=D_{0+}^{q-1} u(t) \in C[0,1] .
$$

By Lemma 3, (8), and $u(t) \in C[0,1]$, one gets

$$
\begin{gathered}
u(t)=I_{0+}^{q} f(t)+\frac{F(0)}{\Gamma(q)} t^{q-1}, \\
I_{0+}^{q-\delta} F(t)=D_{0+}^{\delta-1} u(t) \in C[0,1] .
\end{gathered}
$$

Together with Property 1(3) and the continuity of $F$, taking the limit $\delta \rightarrow q$, we obtain

$$
F(t)=\lim _{\delta \rightarrow q} D_{0+}^{\delta-1} u(t), \quad \forall t \in(0,1] .
$$

Moreover,

$$
F(0)=\lim _{t \rightarrow 0^{+}} F(t), \quad D_{0+}^{\delta-1} u(t)_{\mid t=0}=\lim _{t \rightarrow 0^{+}} D_{0+}^{\delta-1} u(t) .
$$

From (11), (12), and the uniform convergence of $D_{0+}^{\delta-1} u(t)$, it follows that

$$
F(0)=\lim _{\delta \rightarrow q} D_{0+}^{\delta-1} u(t)_{\mid t=0}
$$


Using (9) and Property 1(1)-(2) we obtain that $g_{\delta}(t):=$ $D_{0+}^{\delta} u(t)$ exists and belongs to $L(0,1)$, satisfying

$$
u(t)=I_{0+}^{\delta} g_{\delta}(t)+\frac{D_{0+}^{\delta-1} u(t)_{\mid t=0}}{\Gamma(\delta)} t^{\delta-1}, \quad t \in[0,1]
$$

To the end, it suffices to show that $\lim _{\delta \rightarrow q} g_{\delta}(t)=f(t)$, $t \in(0,1]$. Obviously, it follows from (9) and (14) that

$$
\begin{aligned}
& \int_{0}^{t} \frac{(t-s)^{q-1}}{\Gamma(q)} f(s)-\frac{(t-s)^{\delta-1}}{\Gamma(\delta)} g_{\delta}(s)+\frac{F(0)}{\Gamma(q-1)} s^{q-2} \\
& -\frac{D_{0+}^{\delta-1} u(t)_{\mid t=0}}{\Gamma(\delta-1)} s^{\delta-2} d s=0 .
\end{aligned}
$$

For the homogeneous Abel integral equation (15), we observe that the integrand belongs to $L(0,1)$. Then by Lemma 2.5 in [1]

$$
\begin{aligned}
& \frac{(t-s)^{q-1}}{\Gamma(q)} f(s)-\frac{(t-s)^{\delta-1}}{\Gamma(\delta)} g_{\delta}(s)+\frac{F(0)}{\Gamma(q-1)} s^{q-2} \\
& \quad-\frac{D_{0+}^{\delta-1} u(t)_{\mid t=0}}{\Gamma(\delta-1)} s^{\delta-2} \equiv 0 .
\end{aligned}
$$

Taking the limit $\delta \rightarrow q$ in (16), together with (13) we have

$$
\frac{(t-s)^{q-1}}{\Gamma(q)}\left[f(s)-\lim _{\delta \rightarrow q} g_{\delta}(s)\right] \equiv 0, \quad s \in(0,1] .
$$

Hence $\lim _{\delta \rightarrow q} g_{\delta}(t)=f(t), t \in(0,1]$. The proof is complete.

Remark 5. In fact, if it holds that $u(t) \in C[0,1]$ and that $D_{0+}^{q} u(t) \in C[0,1], q \in(1,2)$, then by Lemma 3 , Property $1(1)$, (3), and $\Gamma(0)=\infty$, it directly follows that $\lim _{\delta \rightarrow q^{-}} D_{0+}^{\delta} u(t)=$ $D_{0+}^{q} u(t), \forall t \in(0,1]$. With the above arguments in Lemma 4 , we easily get the following result.

Lemma 6. Suppose that $u(t) \in C[0,1]$ and $D_{0+}^{q} u(t) \in C[0,1]$, $q \in(0,1)$, then $\lim _{\delta \rightarrow q^{-}} D_{0+}^{\delta} u(t)=D_{0+}^{q} u(t), t \in[0,1]$.

Lemma 7. If $g(t) \in C[0,1]$, then the following fractional boundary value problem

$$
\begin{gathered}
D_{0+}^{q} u(t)+g(t)=0, \quad 0<t<1,1<q<2, \\
u(0)=0, \quad D_{0+}^{q-1} u(t)_{\mid t=1}=\eta,
\end{gathered}
$$

has a unique solution:

$$
u(t)=\int_{0}^{1} G(t, s) g(s) d s+\frac{\eta}{\Gamma(q)} t^{q-1},
$$

where

$$
G(t, s)=\frac{1}{\Gamma(q)} \begin{cases}t^{q-1}-(t-s)^{q-1}, & 0 \leq s \leq t \leq 1 \\ t^{q-1}, & 0 \leq t \leq s \leq 1\end{cases}
$$

The proof is standard; we omit it here.
Remark 8. Obviously, $G(t, s) \in C([0,1] \times[0,1])$ and $G(t, s) \leq$ $t^{q-1} / \Gamma(q),(t, s) \in[0,1] \times[0,1]$.

In [18], Al-Refai obtained the following interesting result concerning the Riemann-Liouville fractional derivative at extreme points, where there is a little mistake. Now we state it correctly without proof.

Theorem 9 (see [18]). Let $u(t) \in C^{2}[0,1]$ attain its global minimum at $t_{0} \in(0,1)$; then

$$
D_{0+}^{\delta} u(t)_{\mid t=t_{0}} \geq \frac{(1-\delta)}{\Gamma(2-\delta)} t_{0}^{-\delta} u\left(t_{0}\right), \quad \forall 1<\delta<2 .
$$

Remark 10. In the sense of Riemann-Liouville fractional derivative of order $1<\delta<2$, in general the assumption that $u \in C^{2}[0,1]$ is difficult to meet due to the fact that the fundamental solution of certain corresponding homogeneous differential equations possesses a singularity at $t=0$. Hence, we hope to weaken the conditions of the above theorem.

In the following, we give some lemmas, wherein some ideas in the proofs come from $[18,19]$, with weaker hypotheses.

Lemma 11. Assume that $u(t) \in C[0,1] \cap C^{1}(0,1]$ satisfies the following conditions:

(i) $D_{0+}^{\delta} u(t)$ exist, $t \in[0,1]$, for $\delta \in(1,2)$;

(ii) there exists constant $\theta>0$, such that $t^{\theta} u^{\prime}(t)$ is Hölder continuous with exponent $\sigma>\delta-1$;

(iii) $u(t)$ attains its global minimum at $t_{0} \in(0,1)$.

Then,

$$
D_{0+}^{\delta} u(t)_{\mid t=t_{0}} \geq \frac{(1-\delta)}{\Gamma(2-\delta)} t_{0}^{-\delta} u\left(t_{0}\right), \quad \forall 1<\delta<2 .
$$

Moreover, if $u\left(t_{0}\right) \leq 0$, then $D_{0+}^{\delta} u(t)_{\mid t=t_{0}} \geq 0$.

Proof. Let $f(t):=u(t)-u\left(t_{0}\right), t \in[0,1]$. Obviously, $f(t) \in$ $C[0,1] \cap C^{1}(0,1]$, satisfying the conditions (i)-(iii). It follows that

$$
\begin{gathered}
f^{\prime}(t)=u^{\prime}(t), \quad f(t) \geq 0, \quad t \in[0,1] \\
f\left(t_{0}\right)=f^{\prime}\left(t_{0}\right)=0, \\
D_{0+}^{\delta} f(t)=D_{0+}^{\delta} u(t)-\frac{1-\delta}{\Gamma(2-\delta)} t^{-\delta} u\left(t_{0}\right), \quad t \in[0,1] .
\end{gathered}
$$

Since $f \in C[0,1] \cap C^{1}(0,1]$, we know that $f(t)=f(0)+$ $\int_{0}^{t} f^{\prime}(s) d s, t \in(0,1]$. Then for $1<\delta<2$, it follows from proofs of Lemma 2.1 in [1] that

$$
\begin{aligned}
H(t) & :=D_{0+}^{\delta-1} f(t) \\
& =\frac{f(0)}{\Gamma(2-\delta)} t^{1-\delta}+\frac{1}{\Gamma(2-\delta)} \int_{0}^{t} \frac{f^{\prime}(s)}{(t-s)^{\delta-1}} d s,
\end{aligned}
$$


At this point, we choose enough small constant $r$, such that $0<r<1-t_{0}$. Since $D_{0+}^{\delta} f(t)$ exists, $D_{0+}^{\delta} f(t)_{\mid t=t_{0}}=$ $H^{\prime}\left(t_{0}\right)=\lim _{r \rightarrow 0^{+}}\left(\left(H\left(t_{0}+r\right)-H\left(t_{0}\right)\right) / r\right)$. We consider

$$
\begin{aligned}
& H\left(t_{0}+r\right)-H\left(t_{0}\right) \\
& =\frac{1}{\Gamma(2-\delta)}\left\{\int_{0}^{t_{0}}\left[\left(t_{0}+r-s\right)^{1-\delta}-\left(t_{0}-s\right)^{1-\delta}\right] f^{\prime}(s) d s\right. \\
& \left.\quad+\int_{t_{0}}^{t_{0}+r}\left(t_{0}+r-s\right)^{1-\delta} f^{\prime}(s) d s\right\} \\
& \quad+\frac{f(0)}{\Gamma(2-\delta)}\left[\left(t_{0}+r\right)^{1-\delta}-t_{0}^{1-\delta}\right] .
\end{aligned}
$$

It obviously follows from $f \in C^{1}\left[t_{0}, 1\right]$ that the second integration in (26) converges, and then the first integration in (26) also converges. For the latter, applying the integration by parts, together with (23), we have

$$
\begin{aligned}
\int_{0}^{t_{0}}[ & \left.\left(t_{0}+r-s\right)^{1-\delta}-\left(t_{0}-s\right)^{1-\delta}\right] f^{\prime}(s) d s \\
= & \lim _{s \rightarrow t_{0}^{-}}\left[\left(t_{0}+r-s\right)^{1-\delta}-\left(t_{0}-s\right)^{1-\delta}\right] f(s) \\
& -\lim _{s \rightarrow 0^{+}}\left[\left(t_{0}+r-s\right)^{1-\delta}-\left(t_{0}-s\right)^{1-\delta}\right] f(s) \\
& +(1-\delta) \int_{0}^{t_{0}}\left[\left(t_{0}+r-s\right)^{-\delta}-\left(t_{0}-s\right)^{-\delta}\right] f(s) d s \\
= & r^{1-\delta} f\left(t_{0}\right)-\lim _{s \rightarrow t_{0}^{-}} \frac{f(s)}{\left(t_{0}-s\right)^{\delta-1}} \\
& -\left[\left(t_{0}+r\right)^{1-\delta}-t_{0}^{1-\delta}\right] f(0) \\
& +(1-\delta) \int_{0}^{t_{0}}\left[\left(t_{0}+r-s\right)^{-\delta}-\left(t_{0}-s\right)^{-\delta}\right] f(s) d s \\
= & \lim _{s \rightarrow t_{0}^{-}} \frac{f\left(t_{0}\right)-f(s)}{t_{0}-s}\left(t_{0}-s\right)^{2-\delta} \\
& +(1-\delta) \int_{0}^{t_{0}}\left[\left(t_{0}+r-s\right)^{-\delta}-\left(t_{0}-s\right)^{-\delta}\right] f(s) d s \\
\geq & -\left[\left(t_{0}+r\right)^{1-\delta}-t_{0}^{1-\delta}\right] f(0), \\
= & -\left[\left(t_{0}+r\right)^{1-\delta}-t_{0}^{1-\delta}\right] f(0) \\
& -\left[\left(t_{0}+r\right)^{1-\delta}-t_{0}^{1-\delta}\right] f(0) \\
t_{0} & {\left[\left(t_{0}+r-s\right)^{-\delta}-\left(t_{0}-s\right)^{-\delta}\right] f(s) d s }
\end{aligned}
$$

due to the fact $\delta \in(1,2), f(t) \geq 0$, and $\left(t_{0}+r-s\right)^{-\delta}<\left(t_{0}-s\right)^{-\delta}$.
Hence (26) yields that

$$
H\left(t_{0}+r\right)-H\left(t_{0}\right) \geq \frac{1}{\Gamma(2-\delta)} \int_{t_{0}}^{t_{0}+r}\left(t_{0}+r-s\right)^{1-\delta} f^{\prime}(s) d s .
$$

From the Hölder continuity of $t^{\theta} f^{\prime}(t)$ on $[0,1]$ for some $\theta>0$, it follows that there exists constant $K>0$, such that

$$
\begin{array}{r}
\left|t^{\theta} f^{\prime}(t)\right|=\left|t^{\theta} f^{\prime}(t)-t_{0}^{\theta} f^{\prime}\left(t_{0}\right)\right| \leq K\left(t-t_{0}\right)^{\sigma}, \\
t \in\left[t_{0}, 1\right], \quad \sigma>\delta-1 .
\end{array}
$$

For $t \in\left[t_{0}, t_{0}+r\right] \subset\left[t_{0}, 1\right]$, we have $\left|f^{\prime}(t)\right| \leq K t^{-\theta}\left(t-t_{0}\right)^{\sigma} \leq$ $K t_{0}^{-\theta} r^{\sigma}$. Thus,

$$
\begin{aligned}
H\left(t_{0}+r\right)-H\left(t_{0}\right) & \geq \frac{-K t_{0}^{-\theta} r^{\sigma}}{\Gamma(2-\delta)} \int_{t_{0}}^{t_{0}+r}\left(t_{0}+r-s\right)^{1-\delta} d s \\
& =\frac{-K t_{0}^{-\theta}}{\Gamma(3-\delta)} r^{2+\sigma-\delta} .
\end{aligned}
$$

Dividing by $r$ on both sides of (30) and taking the limit $r \rightarrow$ 0 , one gets

$$
\begin{aligned}
D_{0+}^{\delta} f(t)_{\mid t=t_{0}} & =\lim _{r \rightarrow 0^{+}} \frac{H\left(t_{0}+r\right)-H\left(t_{0}\right)}{r} \\
& \geq \lim _{r \rightarrow 0^{+}} \frac{-K t_{0}^{\delta-2}}{\Gamma(3-\delta)} r^{1+\sigma-\delta}=0 .
\end{aligned}
$$

Together with (24), we obtain

$$
D_{0+}^{\delta} u(t)_{\mid t=t_{0}} \geq \frac{1-\delta}{\Gamma(2-\delta)} t_{0}^{-\delta} u\left(t_{0}\right), \quad t_{0} \in(0,1) .
$$

The proof is complete.

Remark 12. Lemma 11 is an essential improvement of Theorem 9 and crucial for our main theorems. By applying the above results on $-u(t)$, analogous results for RiemannLiouville fractional derivatives at global maximum points are derived. It is worth mentioning that the weaker requirement $\sigma>\delta-1$ in (ii) seems to be unsatisfactory as well, since in general the exponent $\sigma$ may be guaranteed only up to $\delta-1$. To solve the difficulty, the ideas of reducing the order and approach method are employed in our main theorems.

Spontaneously for the case $0<\delta<1$, Lakshmikantham and Vatsala in [19] established the following result concerning Riemann-Liouville fractional derivative.

Theorem 13 (see [19]). Let $u(t): \mathbb{R}_{+} \rightarrow \mathbb{R}$ be locally Hölder continuous such that for any $t_{0} \in(0, \infty)$, we have

$$
u\left(t_{0}\right)=0, \quad u(t) \leq 0, \quad \text { for } 0 \leq t \leq t_{0} .
$$

Then it follows that

$$
D_{0+}^{\delta} u\left(t_{0}\right) \geq 0, \quad 0<\delta<1 .
$$


Remark 14. As the literature [20] points out, in general, the function $u(t)$ containing term $t^{\delta-1}, \delta \in(0,1)$ is not locally Hölder continuous of any order. For this reason, in [20,21] the authors attempt to weaken the locally Hölder continuity to $C_{1-\delta}$ continuity on $u(t)$; nevertheless, their arguments seem to be flawed as well. Similarl to the proof of Lemma 11, we get the following lemma.

Lemma 15. Assume that $u(t) \in C(0,1]$ satisfies the following conditions:

(i) $D_{0+}^{\delta} u(t)$ exist, $t \in[0,1]$, for $\delta \in(0,1)$;

(ii) there exists constant $\theta \geq 0$, such that $t^{\theta} u(t)$ is Hölder continuous with exponent $\sigma>\delta$;

(iii) $u(t)$ attains its global minimum at $t_{0} \in(0,1]$.

Then,

$$
D_{0+}^{\delta} u(t)_{\mid t=t_{0}} \leq \frac{t_{0}^{-\delta}}{\Gamma(1-\delta)} u\left(t_{0}\right), \quad \forall 0<\delta<1 .
$$

Moreover, if $u\left(t_{0}\right) \leq 0$, then $D_{0+}^{\delta} u(t)_{\mid t=t_{0}} \leq 0$.

Remark 16. Note that in the case $\delta \in(0,1)$, we allow $u(t)$ to attain the minimum at the endpoint $t_{0}=1$. In fact, analogous result for Lemma 15 at global maximum points is the generalization of Theorem 13 .

Recently, some results, for instance, Lemma 2.13 in [22] and Property 4 [23] concerning Hölder continuity for Riemann-Liouville fractional integral operator have been obtained. Therein Bourdin in [23] has proved that fractional integral operator $I_{0+}^{\delta}, \delta \in(0,1)$ maps $L_{p}$ functions to Hölder continuous functions, the exponent of which depends on $\delta$ and $p$. In the following, we give some other results about Hölder continuity.

Lemma 17. Let $0<\delta<1, f(t) \in C[0,1]$. Then $I_{0+}^{\delta} f(t)$ is Hölder continuous with exponent $\delta$ on $[0,1]$.

Proof. Similar to the proof of Property 4 [23], Lemma 17 is easily obtained. So we omit the proof.

Lemma 18 (see [24]). Suppose that $0<a \leq t_{1} \leq t_{2} \leq \xi$, $0<\theta<1$; then,

$$
\left(t_{1}+t_{2}\right)^{\theta} \leq t_{1}^{\theta}+t_{2}^{\theta}
$$

Remark 19. Obviously, Lemma 18 is valid under the assumptions $0 \leq t_{1} \leq t_{2} \leq \xi, 0<\theta<1$.

Lemma 20. Let $f(t)$ be Hölder continuous with exponent $\delta \in$ $(0,1]$ on $[0,1]$. Then $t^{\theta} f(t), \theta>0$ is Hölder continuous with exponent $\min \{\delta, \theta\}$ on $[0,1]$.

Proof. From the Hölder continuity of $f$ on $[0,1]$, it obviously follows that there exist some $L_{1}, L_{2}>0$, such that $|f(t)| \leq L_{1}$ and

$$
\left|f\left(t_{1}\right)-f\left(t_{2}\right)\right| \leq L_{2}\left|t_{1}-t_{2}\right|^{\delta}, \quad \forall t_{1}, t_{2} \in[0,1] .
$$

Without loss of generality, let $t_{1}>t_{2}$. Then,

$$
\begin{aligned}
& \left|t_{1}^{\theta} f\left(t_{1}\right)-t_{2}^{\theta} f\left(t_{2}\right)\right| \\
& \quad=\left|t_{1}^{\theta} f\left(t_{1}\right)-t_{1}^{\theta} f\left(t_{2}\right)+t_{1}^{\theta} f\left(t_{2}\right)-t_{2}^{\theta} f\left(t_{2}\right)\right| \\
& \quad \leq t_{1}^{\theta}\left|f\left(t_{1}\right)-f\left(t_{2}\right)\right|+\left|f\left(t_{2}\right)\right|\left|t_{1}^{\theta}-t_{2}^{\theta}\right| \\
& \quad \leq L_{2}\left(t_{1}-t_{2}\right)^{\delta}+L_{1}\left(t_{1}^{\theta}-t_{2}^{\theta}\right) .
\end{aligned}
$$

If $0<\theta<1$, using Lemma 18 we have

$$
\begin{array}{r}
\left|t_{1}^{\theta} f\left(t_{1}\right)-t_{2}^{\theta} f\left(t_{2}\right)\right| \leq L_{3}\left(t_{1}-t_{2}\right)^{\min \{\delta, \theta\}}, \\
\text { for some } L_{3}>0 .
\end{array}
$$

If $\theta \geq 1$, using the mean value theorem we have

$$
\left|t_{1}^{\theta} f\left(t_{1}\right)-t_{2}^{\theta} f\left(t_{2}\right)\right| \leq L_{4}\left(t_{1}-t_{2}\right)^{\delta}
$$

$$
\text { for some } L_{4}>0 \text {. }
$$

The proof is complete.

Combining Lemmas 17 and 20, we have the following corollary.

Corollary 21. Let $0<\delta<1, f(t) \in C[0,1]$. Then $t^{\theta} I_{0+}^{\delta} f(t)$, $\theta>0$ is Hölder continuous with exponent $\min \{\theta, \delta\}$ on $[0,1]$.

Now we introduce the upper and lower solutions of FBVP (3).

Definition 22. A function $\alpha(t) \in C[0,1] \cap C^{1}(0,1]$, satisfying $D_{0+}^{q} \alpha(t) \in L(0,1)$ with $t^{\theta} \alpha^{\prime}(t) \in H_{0}^{q-1}[0,1], \forall \theta \geq 1$, is called a lower solution of FBVP (3), if it satisfies

$$
\begin{array}{r}
D_{0+}^{q} \alpha(t)+f\left(t, \alpha(t), D_{0+}^{q-1} \alpha(t)\right) \geq 0, \\
t \in[0,1], \quad 1<q<2, \\
\alpha(0)=0, \quad D_{0+}^{q-1} \alpha(t)_{\mid t=1} \leq \eta .
\end{array}
$$

Analogously, a function $\beta(t) \in C[0,1] \cap C^{1}(0,1]$, satisfying $D_{0+}^{q} \beta(t) \in L(0,1)$ with $t^{\theta} \beta^{\prime}(t) \in H_{0}^{q-1}[0,1], \forall \theta \geq 1$, is called an upper solution of FBVP (3), if it satisfies (41)-(42) with reversed inequalities.

Definition 23. Given a pair of functions $\alpha(t), \beta(t)$ satisfying $\alpha(t) \leq \beta(t)$. A function $f:[0,1] \times \mathbb{R}^{2} \rightarrow \mathbb{R}$ is said to satisfy the Nagumo condition with respect to $\alpha(t)$ and $\beta(t)$, if there exists a function $\Phi \in C([0, \infty),(0, \infty))$ such that

$$
|f(t, u, v)| \leq \Phi(|v|),
$$

for all $(t, u, v) \in[0,1] \times[\alpha(t), \beta(t)] \times \mathbb{R}$, and

$$
\int_{0}^{+\infty} \frac{s}{\Phi(s)} d s=+\infty .
$$




\section{Main Results}

In this section, we will apply the method of upper and lower solutions combined with fixed point theorem to consider the existence of solutions of FBVP (3).

Denote by $H_{0}^{\delta}[0,1]$ the set consisting of the Hölder continuous functions $u(t)$ with exponent $\delta \in(0,1]$ on $[0,1]$ and $u(0)=0$.

In this paper, we consider the Banach space $E$ defined by

$$
E=\left\{u(t) \in C[0,1]: D_{0+}^{q-1} u(t) \in C[0,1]\right\},
$$

with the norm $\|u(t)\|=\max _{0 \leq t \leq 1}|u(t)|+\max _{0 \leq t \leq 1}\left|D_{0+}^{q-1} u(t)\right|$. In fact, by Lemmas 3 and 17 it is derived that $E \subset H_{0}^{q-1}[0,1]$.

The main results in this paper are the following.

Theorem 24. Assume that the following conditions hold:

$\left(\mathrm{H}_{1}\right)$ FBVP (3) has a pair of upper and lower solutions $\beta(t), \alpha(t)$, respectively, with

$$
\alpha(t) \leq \beta(t), \quad t \in[0,1]
$$

$\left(\mathrm{H}_{2}\right) f(t, u, v) \in C\left([0,1] \times \mathbb{R}^{2}, \mathbb{R}\right)$ is nonincreasing with respect to the third variable;

$\left(\mathrm{H}_{3}\right) f$ satisfies the Nagumo condition with respect to $\alpha(t)$ and $\beta(t)$.

Then FBVP (3) has at least one solution $u(t) \in C[0,1]$ with $D_{0+}^{q-1} u(t) \in C[0,1]$, such that

$$
\alpha(t) \leq u(t) \leq \beta(t), \quad t \in[0,1] .
$$

Proof. From the assumptions $\left(\mathrm{H}_{1}\right)$ and $\left(\mathrm{H}_{3}\right)$, we know

$$
\lambda:=\max _{0 \leq t \leq 1} I_{0+}^{2-q} \beta(t)-\min _{0 \leq t \leq 1} I_{0+}^{2-q} \alpha(t) \geq 0,
$$

due to the monotonicity of Riemann-Liouville fractional integral operator $I_{0+}^{2-q}$. And choose constant $C>\lambda$, such that

$$
\int_{\lambda}^{C} \frac{s}{\Phi(s)} d s>\lambda .
$$

It is easy to obtain that $\alpha(t), \beta(t) \in E$. We let

$$
L \geq \max \left\{\max _{0 \leq t \leq 1}\left|D_{0+}^{q-1} \beta(t)\right|, \max _{0 \leq t \leq 1}\left|D_{0+}^{q-1} \alpha(t)\right|, C,|\eta|\right\},
$$

and consider the following modified fractional boundary value problem:

$$
\begin{gathered}
D_{0+}^{q} u(t)+F\left(t, u(t), D_{0+}^{q-1} u(t)\right)=0, \quad t \in[0,1], \\
u(0)=0, \quad D_{0+}^{q-1} u(1)=\eta,
\end{gathered}
$$

where

$$
\begin{gathered}
F(t, u, v)= \begin{cases}f_{1}(t, \alpha(t), v), & u<\alpha(t), t \in[0,1], \\
f_{1}(t, u, v), & \alpha(t) \leq u \leq \beta(t), t \in[0,1], \\
f_{1}(t, \beta(t), v), & u>\beta(t), t \in[0,1],\end{cases} \\
f_{1}(t, u, v)= \begin{cases}f(t, u,-L), & v<-L, t \in[0,1], \\
f(t, u, v), & -L \leq v \leq L, t \in[0,1], \\
f(t, u, L), & v>L, t \in[0,1] .\end{cases}
\end{gathered}
$$

Obviously, $F(t, u, v) \quad \in \quad C\left([0,1] \times \mathbb{R}^{2}, \mathbb{R}\right)$ is bounded; that is, there exists positive constant $M \geq$ $\max _{0 \leq t \leq 1, \alpha(t) \leq u \leq \beta(t),-L \leq v \leq L}|f(t, u, v)|$, such that

$$
|F(t, u, v)| \leq M, \quad(t, u, v) \in[0,1] \times \mathbb{R}^{2} .
$$

To the end, it is sufficient to show that the modified FBVP (51)-(52) has at least one solution $u(t)$, satisfying

$$
\alpha(t) \leq u(t) \leq \beta(t), \quad\left|D_{0+}^{q-1} u(t)\right| \leq L, \quad t \in[0,1] .
$$

We divide the proof into three steps.

Step 1. FBVP (51)-(52) has at least one solution $u(t) \in C[0,1]$ with $D_{0+}^{q-1} u(t) \in C[0,1]$.

Firstly, we define the operator $T: E \rightarrow E$ by

$$
\begin{array}{r}
T u(t)=\int_{0}^{1} G(t, s) F\left(s, u(s), D_{0+}^{q-1} u(s)\right) d s+\frac{\eta}{\Gamma(q)} t^{q-1}, \\
t \in[0,1] .
\end{array}
$$

From the continuity of $G(t, s)$ and $F$, it is not difficult to verify that the operator $T: E \rightarrow E$ is well defined and continuous.

By Lemma 7 we can see that the fixed points of $T$ coincide with the solutions of FBVP (51)-(52). In the following, we prove that $T$ has a fixed point in $E$.

Secondly, since $D_{0+}^{q-1} T u(t)=\int_{t}^{1} F\left(s, u(s), D_{0+}^{q-1} u(s)\right) d s+\eta$, $F$ is bounded by $M$, and $G(t, s) \in C([0,1] \times[0,1])$, together with (56) we easily obtain that $T \Omega$ is uniformly bounded (here, $\Omega$ is a bounded subset of $E$ ).

For $\forall u \in \Omega, t_{1}, t_{2} \in[0,1]$, without loss of generality, let $t_{1}>t_{2}$. We have

$$
\begin{aligned}
& \left|T u\left(t_{1}\right)-T u\left(t_{2}\right)\right| \\
& \leq \int_{0}^{1}\left|G\left(t_{1}, s\right)-G\left(t_{2}, s\right)\right|\left|F\left(s, u(s), D_{0+}^{q-1} u(s)\right)\right| d s \\
& \quad+\frac{|\eta|}{\Gamma(q)}\left|t_{1}^{q-1}-t_{2}^{q-1}\right| \\
& \leq \frac{M+|\eta|}{\Gamma(q)}\left(t_{1}^{q-1}-t_{2}^{q-1}\right)+\frac{M}{\Gamma(q+1)}\left(t_{1}^{q}-t_{2}^{q}\right) \\
& \quad \longrightarrow 0, \quad t_{1} \longrightarrow t_{2}, \\
& \left|D_{0+}^{q-1} T u(t)_{\mid t=t_{1}}-D_{0+}^{q-1} T u(t)_{\mid t=t_{2}}\right| \leq M\left(t_{1}-t_{2}\right) \\
& \quad \longrightarrow 0, \quad t_{1} \longrightarrow t_{2} .
\end{aligned}
$$

That is, $T \Omega$ is equicontinuous. According to the Ascoli-Arzela theorem, we know that $T: E \rightarrow E$ is completely continuous.

By the Schauder fixed point theorem, we can easily obtain that $T$ has at least one fixed point $u(t) \in C[0,1]$ with $D_{0+}^{q-1} u(t) \in C[0,1]$.

Step 2. The function $u(t) \in E$ satisfies $\alpha(t) \leq u(t) \leq \beta(t)$, $t \in[0,1]$. 
Suppose that $u(t) \geq \alpha(t)$ on $[0,1]$ is not true; then $w(t):=$ $u(t)-\alpha(t)$ has a negative minimum at some $t_{0} \in[0,1]$; that is, $w\left(t_{0}\right)=\min \{u(t)-\alpha(t) \mid t \in[0,1]\}<0$.

If $t_{0}=0$, then $u(0)<\alpha(0)$. From (42) and (52) we have the contradiction $\alpha(0)=u(0)=0$.

If $t_{0} \in(0,1)$, that is, $u\left(t_{0}\right)<\alpha\left(t_{0}\right)$. Obviously, $w(t) \in$ $C[0,1], D_{0+}^{q} w(t)=D_{0+}^{q} u(t)-D_{0+}^{q} \alpha(t) \in L(0,1)$, which deduces $D_{0+}^{q-1} w(t) \in C[0,1]$. Choosing enough small $p \in$ $(0, q-1)$, by Lemma 4 we obtain that $D_{0+}^{q-p} w(t)$ exists, $t \in[0,1]$ and

$$
\lim _{p \rightarrow 0} D_{0+}^{q-p} w(t)=D_{0+}^{q} w(t), \quad t \in(0,1]
$$

Now, denote $F\left(\cdot, u(\cdot), D_{0+}^{q-1} u(\cdot)\right)$, for brevity, by $F$. From (56) it follows that

$$
u(t)=\frac{\int_{0}^{1} F(s) d s+\eta}{\Gamma(q)} t^{q-1}-I_{0+}^{q} F(t) \in C[0,1] .
$$

Choosing $\theta=3-q>1$ in Lemma 11, then

$$
t^{\theta} u^{\prime}(t)=t^{3-q} u^{\prime}(t)=\frac{\int_{0}^{1} F(s) d s+\eta}{\Gamma(q-1)} t-t^{3-q} I_{0+}^{q-1} F(t) .
$$

Obviously, the first term on the right side of (60) is Lipschitz continuous in $t \in[0,1]$. For the second term on the right side of (60), by the continuity of $F$ and Corollary 21 we obtain $t^{3-q} I_{0+}^{q-1} F(t) \in H_{0}^{q-1}[0,1]$. Thus, it is deduced that $t^{3-q} u^{\prime}(t) \in$ $H_{0}^{q-1}[0,1]$.

On account of $\alpha(t) \in C[0,1] \cap C^{1}(0,1]$ and $t^{3-q} \alpha^{\prime}(t) \epsilon$ $H_{0}^{q-1}[0,1]$, we have $w(t) \in C[0,1] \cap C^{1}(0,1]$ and $t^{3-q} w^{\prime}(t)$ is Hölder continuous with exponent $q-1>q-p-1$. At this point, by Lemma 11 we obtain

$$
D_{0+}^{q-p} w(t)_{\mid t=t_{0}} \geq \frac{1-q+p}{\Gamma(2-q+p)} t_{0}^{p-q} w\left(t_{0}\right), \quad 1<q-p<2 .
$$

Taking the limit $p \rightarrow 0$ in (61), it follows by (58) and $w\left(t_{0}\right)<$ 0 that

$$
D_{0+}^{q} w(t)_{\mid t=t_{0}} \geq \frac{1-q}{\Gamma(2-q)} t_{0}^{-q} w\left(t_{0}\right)>0 .
$$

On the other hand, firstly we claim that it holds that $D_{0+}^{q-1} u(t)_{\mid t=t_{0}}<D_{0+}^{q-1} \alpha(t)_{\mid t=t_{0}}$. It obviously follows that $w(t) \epsilon$ $E$. Then by Lemma 6 we know

$$
\lim _{p \rightarrow 0} D_{0+}^{q-1-p} w(t)=D_{0+}^{q-1} w(t), \quad t \in[0,1] .
$$

Analogously with above arguments for (62), by Lemma 15 and (63) it is not hard to obtain that

$$
D_{0+}^{q-1} w(t)_{\mid t=t_{0}} \leq \frac{t_{0}^{1-q}}{\Gamma(2-q)} w\left(t_{0}\right)<0, \quad 0<q-1<1 .
$$

That is, $D_{0+}^{q-1} u(t)_{\mid t=t_{0}}<D_{0+}^{q-1} \alpha(t)_{\mid t=t_{0}}$. The claim is proved. Again together with $\left(\mathrm{H}_{1}\right),\left(\mathrm{H}_{2}\right)$, and $-L \leq D_{0+}^{q-1} \alpha(t)_{\mid t=t_{0}} \leq L$, for $u\left(t_{0}\right)<\alpha\left(t_{0}\right)$, we have the following two cases.

Case 1. When $-L \leq D_{0+}^{q-1} u(t)_{\mid t=t_{0}} \leq L$,

$$
\begin{aligned}
D_{0+}^{q} w(t)_{\mid t=t_{0}}= & D_{0+}^{q} u(t)_{\mid t=t_{0}}-D_{0+}^{q} \alpha(t)_{\mid t=t_{0}} \\
\leq & -F\left(t_{0}, u\left(t_{0}\right), D_{0+}^{q-1} u(t)_{\mid t=t_{0}}\right) \\
& +f\left(t_{0}, \alpha\left(t_{0}\right), D_{0+}^{q-1} \alpha(t)_{\mid t=t_{0}}\right) \\
= & -f\left(t_{0}, \alpha\left(t_{0}\right), D_{0+}^{q-1} u(t)_{\mid t=t_{0}}\right) \\
& +f\left(t_{0}, \alpha\left(t_{0}\right), D_{0+}^{q-1} \alpha(t)_{\mid t=t_{0}}\right)
\end{aligned}
$$

$\leq 0$,

which contradicts (62).

Case 2. When $D_{0+}^{q-1} u(t)_{\mid t=t_{0}}<-L$,

$$
\begin{aligned}
D_{0+}^{q} w(t)_{\mid t=t_{0}}= & D_{0+}^{q} u(t)_{\mid t=t_{0}}-D_{0+}^{q} \alpha(t)_{\mid t=t_{0}} \\
\leq & -F\left(t_{0}, u\left(t_{0}\right), D_{0+}^{q-1} u(t)_{\mid t=t_{0}}\right) \\
& +f\left(t_{0}, \alpha\left(t_{0}\right), D_{0+}^{q-1} \alpha(t)_{\mid t=t_{0}}\right) \\
= & -f\left(t_{0}, \alpha\left(t_{0}\right),-L\right) \\
& +f\left(t_{0}, \alpha\left(t_{0}\right), D_{0+}^{q-1} \alpha(t)_{\mid t=t_{0}}\right) \\
\leq & 0,
\end{aligned}
$$

which contradicts (62).

Thus, we know that the minimum point $t_{0}$ satisfying $u(t)<\alpha(t)$ does not occur on $(0,1)$.

If $t_{0}=1$, that is, $w(1)<0$. By the boundary conditions (42) and (52), we have

$$
D_{0+}^{q-1} w(t)_{\mid t=1}=D_{0+}^{q-1} u(t)_{\mid t=1}-D_{0+}^{q-1} \alpha(t)_{\mid t=1} \geq 0 .
$$

However, analogously with above arguments for (64) we obtain

$$
D_{0+}^{q-1} w(t)_{\mid t=1} \leq \frac{w(1)}{\Gamma(2-q)}<0, \quad 0<q-1<1,
$$

which is a contradiction.

Then it holds that $u(t) \geq \alpha(t), t \in[0,1]$. Analogously we can also obtain that $u(t) \leq \beta(t), t \in[0,1]$. Hence, we have $\alpha(t) \leq u(t) \leq \beta(t), t \in[0,1]$.

Step 3. We prove that $\left|D_{0+}^{q-1} u(t)\right| \leq L$ on $[0,1]$.

We only need to show that $D_{0+}^{q-1} u(t) \leq L$ on $[0,1]$. Similarly we can show that $D_{0+}^{q-1} u(t) \geq-L$ on $[0,1]$.

Suppose that $D_{0+}^{q-1} u(t) \leq L$ on $[0,1]$ is not true; then there exists $t_{1} \in[0,1]$, satisfying $D_{0+}^{q-1} u(t)_{\mid t=t_{1}}>L$. Due to 
$I_{0+}^{2-q} u(t) \in C^{1}[0,1]$, and $\alpha(t) \leq u(t) \leq \beta(t), t \in[0,1]$, we know that $I_{0+}^{2-q} \alpha(t) \leq I_{0+}^{2-q} u(t) \leq I_{0+}^{2-q} \beta(t)$, and by the mean value theorem, there exists $t_{2} \in(0,1)$, such that

$$
\begin{aligned}
D_{0+}^{q-1} u(t)_{\mid t=t_{2}} & =I_{0+}^{2-q} u(t)_{\mid t=1}-I_{0+}^{2-q} u(t)_{\mid t=0} \\
& \leq \lambda<L<D_{0+}^{q-1} u(t)_{\mid t=t_{1}} .
\end{aligned}
$$

Since $D_{0+}^{q-1} u(t) \in C[0,1]$, there exists an interval $\left[t_{3}, t_{4}\right] \subset$ $[0,1]\left(\right.$ or $\left.\left[t_{4}, t_{3}\right] \subset[0,1]\right)$ such that

$$
\begin{array}{r}
D_{0+}^{q-1} u(t)_{\mid t=t_{3}}=\lambda, \quad D_{0+}^{q-1} u(t)_{\mid t=t_{4}}=L, \\
\lambda<D_{0+}^{q-1} u(t)<L, \\
t \in\left(t_{3}, t_{4}\right) .
\end{array}
$$

Thus, by $\left(\mathrm{H}_{3}\right)$ we have for $t \in\left(t_{3}, t_{4}\right)$,

$$
\begin{aligned}
D_{0+}^{q} u(t) & \leq\left|F\left(t, u(t), D_{0+}^{q-1} u(t)\right)\right|=\left|f\left(t, u(t), D_{0+}^{q-1} u(t)\right)\right| \\
& \leq \Phi\left(\left|D_{0+}^{q-1} u(t)\right|\right) .
\end{aligned}
$$

Then,

$$
\begin{aligned}
\left|\int_{t_{3}}^{t_{4}} \frac{D_{0+}^{q-1} u(t) \cdot D_{0+}^{q} u(t)}{\Phi\left(\left|D_{0+}^{q-1} u(t)\right|\right)} d t\right| & \leq\left|\int_{t_{3}}^{t_{4}} D_{0+}^{q-1} u(t) d t\right| \\
& =\left|I_{0+}^{2-q} u\left(t_{4}\right)-I_{0+}^{2-q} u\left(t_{3}\right)\right| \\
& \leq \lambda .
\end{aligned}
$$

However, by (49)-(50) and Property 1(4) we have

$$
\begin{aligned}
\left|\int_{t_{3}}^{t_{4}} \frac{D_{0+}^{q-1} u(t) \cdot D_{0+}^{q} u(t)}{\Phi\left(\left|D_{0+}^{q-1} u(t)\right|\right)} d t\right| & =\left|\int_{D_{0+}^{q-1} u(t)_{\mid t=t_{3}}}^{D_{0+}^{q-1} u(t)_{\mid t=t_{4}}} \frac{s}{\Phi(s)} d s\right| \\
& =\int_{\lambda}^{L} \frac{s}{\Phi(s)} d s>\lambda,
\end{aligned}
$$

which is a contradiction with (72). So there holds that $D_{0+}^{q-1} u(t) \leq L, t \in[0,1]$. Hence, we have that $\left|D_{0+}^{q-1} u(t)\right| \leq L$, $t \in[0,1]$. that

Consequently, combining Step 2 and Step 3, we obtain

$$
\begin{array}{r}
D_{0+}^{q} u(t)=F\left(t, u(t), D_{0+}^{q-1} u(t)\right)=f\left(t, u(t), D_{0+}^{q-1} u(t)\right), \\
t \in[0,1] .
\end{array}
$$

That is to say, the solution $u(t)$ is a solution of FBVP (3). Then FBVP (3) has at least one solution $u(t) \in C[0,1]$ with $D_{0+}^{q-1} u(t) \in C[0,1]$, such that $\alpha(t) \leq u(t) \leq \beta(t), t \in$ $[0,1]$.

Remark 25. Observe that the validity of the first inequality in (62) can be guaranteed by means of applying approach method. With this idea, combining Lemma 3, Remark 5, Lemma 11, and Corollary 21, under certain stronger conditions instead of (i)-(ii) in Lemma 11, in the end we have two more concise conclusions as follows.

Theorem 26. Assume that $u(t) \in C[0,1]$ satisfies the following conditions:

(i) $D_{0+}^{\delta} u(t) \in C[0,1]$, for $\delta \in(1,2)$;

(ii) $u(t)$ attains its global minimum at $t_{0} \in(0,1)$.

Then,

$$
D_{0+}^{\delta} u(t)_{\mid t=t_{0}} \geq \frac{(1-\delta)}{\Gamma(2-\delta)} t_{0}^{-\delta} u\left(t_{0}\right), \quad \forall 1<\delta<2
$$

Moreover, if $u\left(t_{0}\right) \leq 0$, then $D_{0+}^{\delta} u(t)_{\mid t=t_{0}} \geq 0$.

Remark 27. In fact, $D_{0+}^{\delta} u(t) \in C[0,1]$ with $u(t) \in C[0,1]$ implies $u(t) \in C^{1}(0,1]$. We emphasize that this result may help to establish some fractional comparison principles for the case of order lying in $(1,2)$, which play a very important role in studying Riemann-Liouville fractional differential equations by means of monotone iterative method. Some related studies will be given in a future paper.

Theorem 28. Assume that $u(t) \in C(0,1]$ satisfies the following conditions:

(i) $D_{0+}^{\delta} u(t) \in C[0,1]$, for $\delta \in(0,1)$;

(ii) $u(t)$ attains its global minimum at $t_{0} \in(0,1]$.

Then,

$$
D_{0+}^{\delta} u(t)_{\mid t=t_{0}} \leq \frac{t_{0}^{-\delta}}{\Gamma(1-\delta)} u\left(t_{0}\right), \quad \forall 0<\delta<1
$$

Moreover, if $u\left(t_{0}\right) \leq 0$, then $D_{0+}^{\delta} u(t)_{\mid t=t_{0}} \leq 0$.

\section{Examples}

Example 29. Consider the following fractional boundary value problem:

$$
\begin{gathered}
D_{0+}^{1.5} u(t)-t\left[u(t)+D_{0+}^{0.5} u(t)\right]=0, \quad t \in[0,1] \\
u(0)=0, \quad D_{0+}^{0.5} u(1)=\frac{1}{2} .
\end{gathered}
$$

Let $q=1.5, f(t, u, v)=-t(u+v)$. Obviously, $f \in C([0,1] \times$ $\left.\mathbb{R}^{2}, \mathbb{R}\right)$, and $d f / d v=-t \leq 0, t \in[0,1]$. It follows that $f$ is nonincreasing with respect to $v$. Choose $\alpha(t)=-(2 / \sqrt{\pi}) t^{0.5}$, $t \in[0,1]$, then $\alpha(t) \in C[0,1] \cap C^{1}(0,1], D_{0+}^{1.5} \alpha(t)=0$, and $t^{\theta} \alpha^{\prime}(t)=-(1 / \sqrt{\pi}) t^{\theta-0.5}, \forall \theta \geq 1$, which deduces that $t^{\theta} \alpha^{\prime}(t) \epsilon$ $H_{0}^{0.5}[0,1]$. And it is not difficult to check out that $\alpha(t)$ is a lower solution of FBVP (77). Analogously, $\beta(t)=(2 / \sqrt{\pi}) t^{0.5}$, $t \in[0,1]$ is an upper solution of $\operatorname{FBVP}(77)$. 
Meanwhile, if we choose $\Phi(s)=s+2$, then when $t \in[0,1]$, $-(2 / \sqrt{\pi}) t^{0.5} \leq u \leq(2 / \sqrt{\pi}) t^{0.5}, v \in \mathbb{R}$, it holds that

$$
\begin{gathered}
|f(t, u, v)| \leq \frac{2}{\sqrt{\pi}}+|v| \leq \Phi(|v|), \\
\int_{0}^{+\infty} \frac{s}{\Phi(s)} d s=\int_{0}^{+\infty} \frac{s}{s+2} d s=+\infty .
\end{gathered}
$$

That is, $f$ satisfies the Nagumo condition with respect to $-(2 / \sqrt{\pi}) t^{0.5}$ and $(2 / \sqrt{\pi}) t^{0.5}$.

Hence, by Theorem 24 we have that FBVP (77) has at least one solution $u(t)$, satisfying $-(2 / \sqrt{\pi}) t^{0.5} \leq u(t) \leq(2 / \sqrt{\pi}) t^{0.5}$, $t \in[0,1]$.

\section{Conflict of Interests}

The authors declare that there is no conflict of interests regarding the publication of this paper.

\section{Acknowledgments}

This work is supported by the Scientific Research Fund of Hunan Provincial Education Department (13K029) and the Innovation Fund Project for Graduate Student of Hunan Province (CX2013B219) and partially supported by the National Nature Science Foundation of China (61170320) and Nature Science Foundation of Guangdong Medical College (B2012053).

\section{References}

[1] S. G. Samko, A. A. Kilbas, and O. I. Marichev, Fractional Integrals and Derivatives, Theory and Applications, Gordon and Breach Science Publishers, Yverdon, Switzerland, 1993.

[2] I. Podlubny, Fractional Differential Equations, vol. 198 of Mathematics in Science and Engineering, Academic Press, New York, NY, USA, 1999.

[3] B. Ahmad, J. J. Nieto, A. Alsaedi, and M. El-Shahed, "A study of nonlinear Langevin equation involving two fractional orders in different intervals," Nonlinear Analysis: Real World Applications, vol. 13, no. 2, pp. 599-606, 2012.

[4] A. Cabada and G. Wang, "Positive solutions of nonlinear fractional differential equations with integral boundary value conditions," Journal of Mathematical Analysis and Applications, vol. 389, no. 1, pp. 403-411, 2012.

[5] X. Su, "Boundary value problem for a coupled system of nonlinear fractional differential equations," Applied Mathematics Letters, vol. 22, no. 1, pp. 64-69, 2009.

[6] N. Kosmatov, "A singular boundary value problem for nonlinear differential equations of fractional order," Journal of Applied Mathematics and Computing, vol. 29, no. 1-2, pp. 125-135, 2009.

[7] F. Jiao and Y. Zhou, "Existence of solutions for a class of fractional boundary value problems via critical point theory," Computers \& Mathematics with Applications, vol. 62, no. 3, pp. 11811199, 2011.

[8] J. Chen and X. H. Tang, "Existence and multiplicity of solutions for some fractional boundary value problem via critical point theory," Abstract and Applied Analysis, vol. 2012, Article ID 648635, 21 pages, 2012.
[9] M. Al-Refai and M. A. Hajji, "Monotone iterative sequences for nonlinear boundary value problems of fractional order," Nonlinear Analysis: Theory, Methods \& Applications, vol. 74, no. 11, pp. 3531-3539, 2011.

[10] T. Jankowski, "Fractional problems with advanced arguments," Applied Mathematics and Computation, vol. 230, pp. 371-382, 2014.

[11] S. Zhang, "Existence of a solution for the fractional differential equation with nonlinear boundary conditions," Computers \& Mathematics with Applications, vol. 61, no. 4, pp. 1202-1208, 2011.

[12] G. Wang, "Monotone iterative technique for boundary value problems of a nonlinear fractional differential equation with deviating arguments," Journal of Computational and Applied Mathematics, vol. 236, no. 9, pp. 2425-2430, 2012.

[13] X. Su and S. Zhang, "Unbounded solutions to a boundary value problem of fractional order on the half-line," Computers \& Mathematics with Applications, vol. 61, no. 4, pp. 1079-1087, 2011.

[14] R. P. Agarwal, D. O'Regan, and S. Stanĕk, "Positive solutions for Dirichlet problems of singular nonlinear fractional differential equations," Journal of Mathematical Analysis and Applications, vol. 371, no. 1, pp. 57-68, 2010.

[15] H. Lian, P. Wang, and W. Ge, "Unbounded upper and lower solutions method for Sturm-Liouville boundary value problem on infinite intervals," Nonlinear Analysis: Theory, Methods and Applications, vol. 70, no. 7, pp. 2627-2633, 2009.

[16] R. A. Khan and J. R. L. Webb, "Existence of at least three solutions of a second-order three-point boundary value problem," Nonlinear Analysis: Theory, Methods \& Applications, vol. 64, no. 6, pp. 1356-1366, 2006.

[17] M. R. Grossinho, F. Minhós, and A. I. Santos, "A note on a class of problems for a higher-order fully nonlinear equation under one-sided Nagumo-type condition," Nonlinear Analysis, vol. 70, no. 11, pp. 4027-4038, 2009.

[18] M. Al-Refai, "On the fractional derivatives at extreme points," Electronic Journal of Qualitative Theory of Differential Equations, no. 55, pp. 1-5, 2012.

[19] V. Lakshmikantham and A. S. Vatsala, "Theory of fractional differential inequalities and applications," Communications in Applied Analysis, vol. 11, no. 3-4, pp. 395-402, 2007.

[20] Z. Denton and A. S. Vatsala, "Monotone iterative technique for finite systems of nonlinear Riemann-Liouville fractional differential equations," Opuscula Mathematica, vol. 31, no. 3, pp. 327339, 2011.

[21] J. V. Devi, F. A. McRae, and Z. Drici, "Variational Lyapunov method for fractional differential equations," Computers \& Mathematics with Applications, vol. 64, no. 10, pp. 2982-2989, 2012.

[22] J. D. Ramírez and A. S. Vatsala, "Monotone iterative technique for fractional differential equations with periodic boundary conditions," Opuscula Mathematica, vol. 29, no. 3, pp. 289-304, 2009.

[23] L. Bourdin, "Existence of a weak solution for fractional EulerLagrange equations," Journal of Mathematical Analysis and Applications, vol. 399, no. 1, pp. 239-251, 2013.

[24] Y. Xu and Z. He, "Existence and uniqueness results for Cauchy problem of variable-order fractional differential equations," Journal of Applied Mathematics and Computing, vol. 43, no. 1-2, pp. 295-306, 2013. 


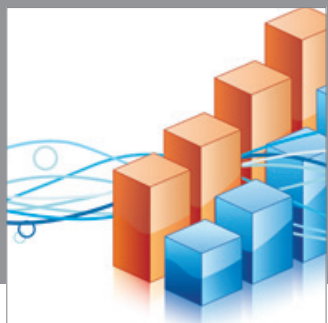

Advances in

Operations Research

mansans

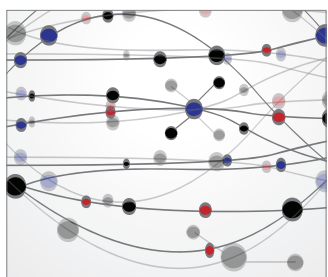

The Scientific World Journal
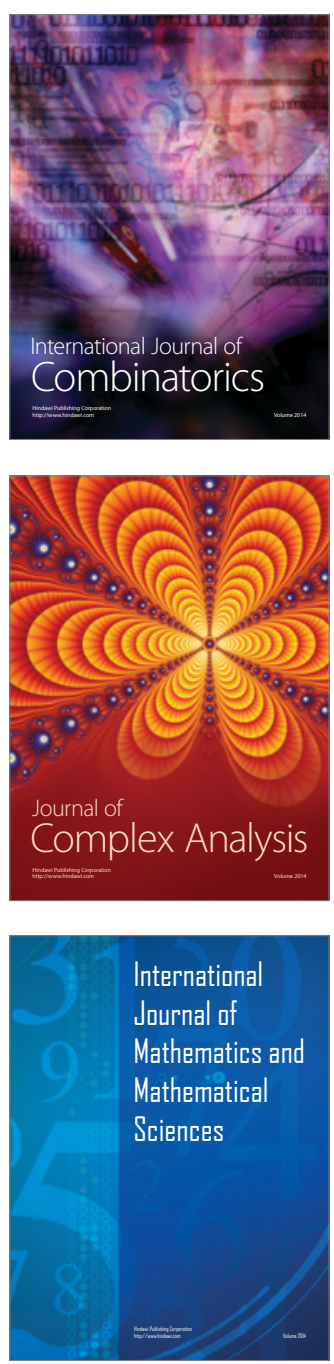
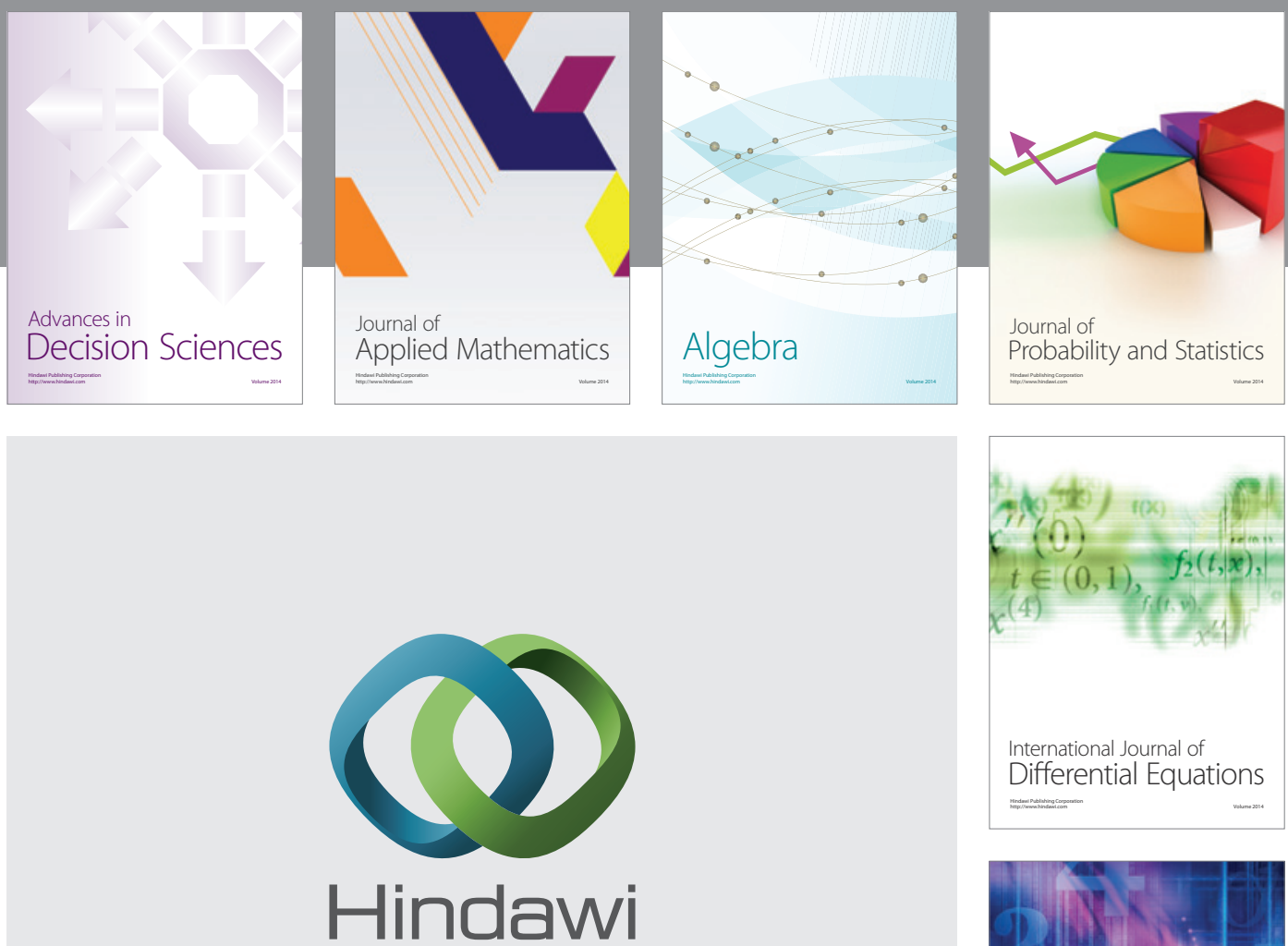

Submit your manuscripts at http://www.hindawi.com
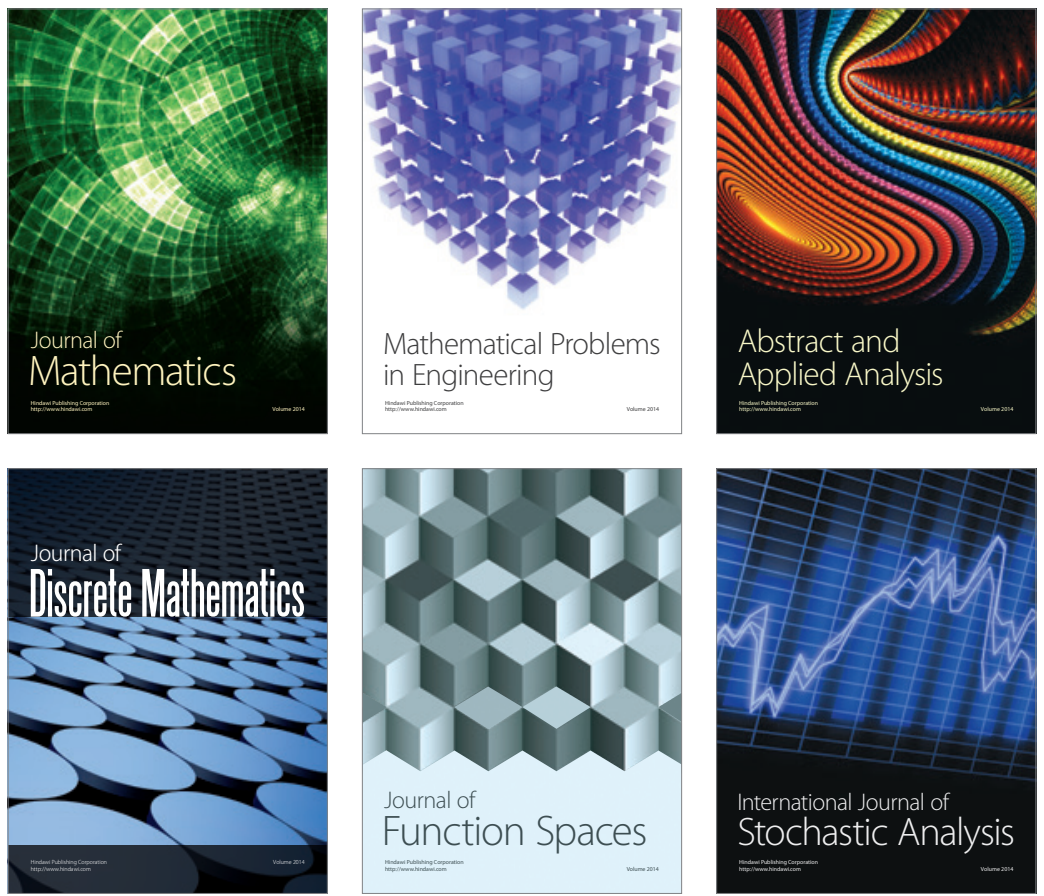

Journal of

Function Spaces

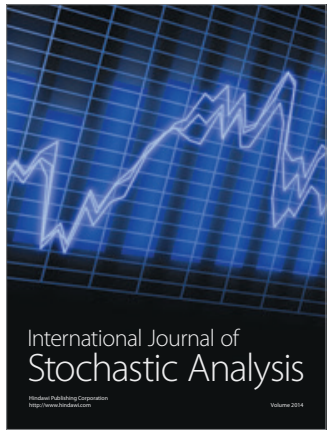

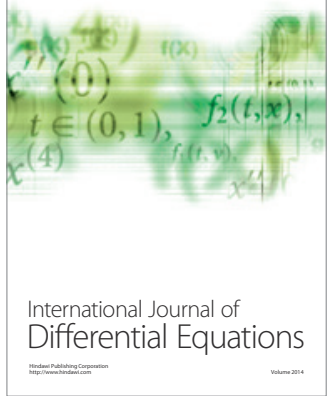
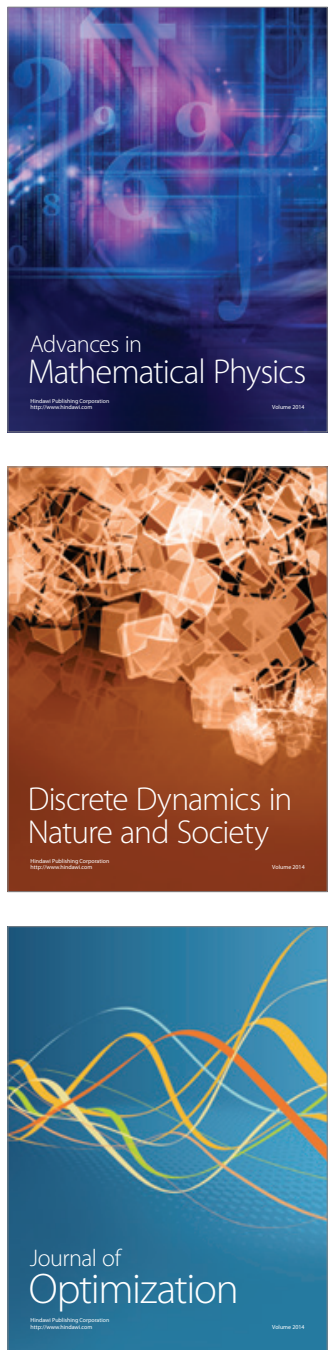